\title{
Pérdida ambigua: madres de personas desaparecidas en Tamaulipas, México
}

\section{Ambiguous Loss: Mothers of Missing Persons in Tamaulipas, Mexico}

\author{
Ariagor Manuel Almanza-Avendaño* (D) http://orcid.org/0000-0001-7240-6163 \\ Ricardo Hernández-Brussolo ** (i) https://orcid.org/0000-0002-7458-9765 \\ Anel Hortensia Gómez-San Luis ${ }^{* * *}$ (1) https://orcid.org/0000-0002-9846-5046
}

\begin{abstract}
Resumen
El objetivo del estudio es comprender la pérdida ambigua de cinco madres de personas que sufrieron desaparición forzada en el estado de Tamaulipas, México. Se realizaron entrevistas en profundidad y se efectuó un análisis fenomenológico-interpretativo. Los resultados muestran que las madres experimentan sufrimiento social y ruptura de la vida cotidiana ante la ausencia física de su hijo y que desarrollan un mecanismo para facilitar su presencia psicológica, aunque siguen experimentando malestar. Entre los elementos del contexto que prolongan el sufrimiento, se encuentran la ineficiencia institucional y el aislamiento social. Una de las limitaciones del estudio es no haber incorporado las voces de otros miembros de la familia, de las madres que han sufrido desplazamiento forzado y de aquellas que no denuncian la desaparición por miedo. Se concluye que es necesario que el Estado reconozca su participación en el origen y en la continuidad del sufrimiento social y que sus intervenciones incorporen la dimensión psicosocial y comunitaria. Palabras clave: madres; desaparición forzada; pérdida ambigua; Tamaulipas.
\end{abstract}

\begin{abstract}
The objective of this research is to understand the ambiguous loss of five mothers of people who suffered forced disappearance in the state of Tamaulipas, Mexico. In-depth interviews were conducted and an interpretative-phenomenological analysis was made. The results show that this mothers experience social suffering and disruption of daily life due to the absence of their child and develop a mechanism to facilitate his or her psychological presence, they but continue experiencing discomfort. Among the context elements that prolong the suffering are the institutional inefficiency and social isolation. One limitation of this research is the failure to incorporate the voices of other family members, those of mothers who have suffered forced displacement, and of those that do not report the disappearance due to fear. It is concluded that it is necessary for the State to recognize its participation in the origin and the maintenance of social suffering and that its interventions must incorporate the psychosocial and community dimension.

Keywords: mothers; forced disappearance; ambiguous loss; Tamaulipas.
\end{abstract}

Cómo citar: Almanza-Avendaño, A. M., Hernández-Brussolo, R., y Gómez-San Luis, A. H. (2020). Pérdida ambigua: madres de personas desaparecidas en Tamaulipas, México. región y sociedad, 32, el 396. doi: 10.22198/rys2020/32/I 396

* Universidad Autónoma de Baja California, Facultad de Ciencias Humanas. Calz. Castellón s. n., Esperanza Conjunto Urbano, C. P. 21350. Mexicali, Baja California, México. Correo electrónico: almanzaa@uabc.edu.mx

** Universidad Autónoma de Ciudad Juárez, Departamento de Ciencias Sociales. Av. Universidad, Av. Heroico Colegio Militar y Chamizal, C. P. 32300. Ciudad Juárez, Chihuahua, México. Correo electrónico: ricardo.brussolo@uacj.mx

*** Autora para correspondencia. Universidad Autónoma de Baja California, Facultad de Ciencias Humanas. Calz. Castellón s. n., Esperanza Conjunto Urbano, C. P. 21350. Mexicali, Baja California, México. Correo electrónico: agomez82@uabc.

Recibido: 8 de septiembre de 2020

Aceptado: 25 de noviembre de 2020

Liberado: 15 de diciembre de 2020

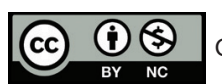

Esta obra está protegida bajo una Licencia

Creative Commons Atribución-No Comercial 4.0 Internacional. 


\section{Introducción}

De acuerdo con la Convención Internacional para la Protección de Todas las Personas contra las Desapariciones Forzadas:

se entenderá por "desaparición forzada" el arresto, la detención, el secuestro o cualquier otra forma de privación de libertad que sean obra de agentes del Estado o por personas o grupos de personas que actúan con la autorización, el apoyo o la aquiescencia del Estado, seguida de la negativa a reconocer dicha privación de libertad o del ocultamiento de la suerte o el paradero de la persona desaparecida, sustrayéndola a la protección de la ley. (Organización de Naciones Unidas, 2006, Art. 2).

El Registro Nacional de Personas Desaparecidas y No Localizadas (RNPDNO) cuenta con un registro histórico -que comprende desde el 15 de marzo de 1964 al 13 de julio de 2002- de 177844 personas desaparecidas, no localizadas y localizadas, de las cuales 73201 (41.16\%) permanecen desaparecidas; 104643 (58.84\%) han sido localizadas, 6401 (6.12\%) localizadas sin vida y 98242 (93.88\%) localizadas con vida. Entre el 1 de diciembre de 2018 y el 13 de julio de 2020, en el sexenio presidencial de Andrés Manual López Obrador, se han registrado 63523 personas desaparecidas, no localizadas y localizadas, de las cuales 27871 (43.88\%) permanecen desaparecidas, 35653 (56.12\%) han sido localizadas, 2352 (6.52\%) localizadas sin vida y 33327 (93.48\%) localizadas con vida (Comisión Nacional de Búsqueda, 2020). La desaparición forzada ha pasado de ser un atentado selectivo que apunta a las individualidades (Deotté, 2015) a ser una catástrofe, como señala Gatti (2011) cuando alude mediante este término a una situación de inestabilidad estable o de excepción permanente, propiciada por la ausencia de instituciones capaces de regular los desajustes sociales.

La desaparición forzada ha superado la figura del desaparecido originario (Gatti, 2017) cuyo victimario es el Estado (Aguayo, 2015). Esta situación ha dado cabida a manifestaciones más complejas e indeterminadas, como los levantados, los encajuelados, los muertos en ácido, los descabezados, los migrantes descuartizados, las mujeres tratadas, asesinadas, violadas o despedazadas. $\mathrm{Ni}$ el Estado ni los agentes paraestatales son necesariamente los responsables directos de estos hechos (Gatti e Irazuzta, 2019). Y es que las nuevas formas de desaparición obedecen a mecanismos de control social y territorial que incluyen el de los flujos migratorios.

Este escenario implica que las fronteras entre el crimen organizado y las fuerzas de seguridad son porosas. Osorno (2017) argumenta que en México existe una necropolítica que dicta quién muere o desaparece en el país, implementada por máquinas de guerra que consisten en grupos especializados para el combate. Estos grupos mantienen diversos tipos de vínculos con el Estado, desde la autonomía hasta la incorporación. La presencia de estas máquinas indica que el Estado ha perdido el monopolio de la violencia y que incluso recurre a la aplicación extrajudicial de ella ante la incapacidad de conservar el orden legal (Benjamin, 1991). 
La región noreste de México, que comprende los estados de Coahuila, Durango, Nuevo León y Tamaulipas, es la más afectada por este delito. En ella se registra un poco más de la tercera parte nacional de los casos (Enciso, 2016). El "Informe especial de la Comisión Nacional de los Derechos Humanos sobre la desaparición de personas y las fosas clandestinas" indica que entre 1995 y agosto de 2015 desaparecieron 5417 personas en Tamaulipas, de las cuales 1303 eran mujeres y 4114 , hombres. De estas personas, 3140 contaban con la mayoría de edad y 633 eran menores de edad. De éstos, 352 eran niñas y 281, niños. Cabe señalar que en 1644 casos no se precisa la edad de las víctimas. De acuerdo con el informe, Tamaulipas, Ciudad de México y el Estado de México son las entidades que presentan mayor incidencia de personas desaparecidas a escala nacional (Comisión Nacional de los Derechos Humanos, 2017).

El aumento de los homicidios y de las desapariciones en Tamaulipas está asociado con factores históricos y coyunturales (Zárate y López, 2017), como la ubicación geográfica, la intensificación del conflicto entre organizaciones rivales, la militarización de las ciudades, la corrupción de funcionarios gubernamentales, el desarrollo de redes macrocriminales entre el gobierno estatal y los grupos del crimen organizado, la deficiencia extrema en la procuración de justicia, la cooptación de las autoridades a escala local, entre otros que han elevado la violencia en el estado a niveles nunca antes vistos, convirtiéndolo en un territorio inaccesible para la sociedad civil (Correa-Cabrera, 2017).

Al principio los grupos criminales en la entidad realizaban actividades como el contrabando, el robo de autos, el control de giros negros o la prostitución, pero durante la década de 1980 se incrementaron las actividades relacionadas con el tráfico de drogas. Durante el periodo presidencial de Carlos Salinas de Gortari (1 de diciembre de 1988 al 30 de noviembre de 1994), el Cártel del Golfo se posicionó como uno de los más poderosos del país. En el sexenio siguiente, el presidente Ernesto Zedillo (1 de diciembre de 1994 al 30 de noviembre de 2000) ordenó la creación de unidades militares especiales para el combate al crimen organizado, de las cuales algunos de sus miembros fueron cooptados por el Cártel del Golfo para cumplir funciones de seguridad y a la postre se convirtieron en los fundadores de Los Zetas (Gibler, 2012). Éstos conformaron una franquicia criminal que se expandió para el control de territorios no sólo de la región noreste, sino también del centro y del sur de México (Ravelo, 2013), en particular en las regiones con abundantes recursos naturales, como hidrocarburos u otros recursos alternativos necesarios para generar energía (Correa-Cabrera, 2018).

Las desapariciones en Tamaulipas no sólo son preocupantes por la cantidad, sino además por el contexto de violencia extrema en el que suceden. El Observatorio sobre Desaparición e Impunidad (ODI, 2019) muestra en el análisis de 166 casos de desaparición, registrados entre 2001 y 2017, que 84\% de éstos fueron eventos colectivos, es decir, que las víctimas fueron capturadas en grupo, lo que sugiere que la desaparición es perpetrada por organizaciones que tienen una estructura criminal compleja, como la de Los Zetas. En la mayoría de los casos no hubo testigos $(79 \%)$ y se desconocen los detalles en que las víctimas fueron capturadas $(76 \%)$. La falta de documentación y de registro de las desapariciones 
puede entenderse por la ausencia de organizaciones de derechos humanos y de la sociedad civil que se deriva de los altos niveles de violencia en el Estado.

Las víctimas de desaparición en Tamaulipas son principalmente varones y mujeres jóvenes en proceso de formación profesional o en edad productiva, aunque los demás grupos de edad también son vulnerables. La entidad se ha convertido en una de las zonas más peligrosas del país para migrantes nacionales y extranjeros (Padgett, 2016). Los lugares más comunes de desaparición son los medios y las vías de transporte (54\%), le siguen los sitios relacionados con la víctima (26\%) y los espacios relacionados con la autoridad (13\%). Es lamentable que la gran mayoría de las personas desaparecidas en Tamaulipas continúen desaparecidas (92\%). Sólo $8 \%$ ha aparecido, de ellos 5\% con vida y 3\% sin vida (ODI, 2019).

Frente a este escenario de desapariciones en Tamaulipas, no es de extrañar la proliferación de fosas clandestinas en espacios abiertos, panteones, ranchos o casas privadas para enterrar los restos de las personas desaparecidas. Incluso, para no dejar rastros, se incineran o se disuelven los cuerpos en lo que el común de la gente denomina cocinas. El contexto de impunidad es sostenido por el Estado mediante la construcción de un marco bajo el que se crea una distinción reduccionista entre víctimas inocentes, sospechosas o culpables, debido a la presunción de asociación o de resistencia al crimen organizado, lo cual impide que cualquier pérdida o ausencia se reconozca como una vida humana insustituible. Tan sólo las víctimas se convierten en una cifra abstracta o en el daño colateral de la estrategia de seguridad (Olalde, 2015; Schedler, 2018). Suponer que las víctimas son inocentes, culpables o sospechosas conlleva el riesgo de olvidar que todas ellas son esencialmente víctimas. Esa conjetura obstaculiza el reconocimiento público del valor de sus vidas e impide la expresión abierta del dolor social que provoca su pérdida.

Las desapariciones tienen serias repercusiones en lo individual, lo colectivo y lo social. La tendencia a enfocarse en el registro de los casos ha limitado el reconocimiento y la atención a las víctimas directas e indirectas de la desaparición. El evento mismo y su contexto provocan consecuencias. La negación por parte del Estado de las víctimas y de sus necesidades, así como las actitudes y los significados sociales que se atribuyen a la desaparición, agudizan su efecto. Las víctimas indirectas suelen desarrollar padecimientos físicos, como estrés crónico, agotamiento, trastornos digestivos, pérdida de peso y problemas cardiovasculares, entre otros. Las afectaciones en la salud mental incluyen crisis de ansiedad, síntomas depresivos, ideación e intento suicida (Retama y Rojas, 2019).

\section{La desaparición como pérdida ambigua}

La desaparición es un estado liminal, caracterizado por la imposibilidad de ser definido socialmente (Robledo, 2016). De acuerdo con Gatti (2011), se trata de una situación singular debido a que hay una disociación entre la identidad y el cuerpo. Un individuo ha perdido su ciudadanía y ha sido expulsado al territorio del afuera; también pierde su nombre, su historia y su espacio. 
Estas condiciones truncan en los familiares el proceso de duelo, pues no hay acceso a la verdad jurídica sobre la desaparición y porque hay incertidumbre sobre el paradero de la persona, lo cual provoca angustia por el riesgo a perderla si está viva, o dolor por creer que ya ha muerto. Cuando no se cuenta con el cuerpo del familiar, se impiden los rituales culturales del funeral, el entierro o las ceremonias religiosas. Sin ellos se restringe la elaboración de la ambivalencia afectiva hacia la persona o la deslibidinización del objeto amado para dirigirse a otros objetos; se paraliza el reconocimiento público de la muerte y la construcción de memoriales para honrar a la persona. Incluso se frena el tránsito de su alma hacia otro mundo, de acuerdo con las creencias de diversos grupos religiosos (Zorio, 2011).

La singularidad de la desaparición forzada requiere el cuestionamiento de algunas nociones tradicionales asociadas con el duelo. Aunque antes el duelo era considerado una reacción normal ante la pérdida de un ser querido, se cree ahora que existen patologías del duelo, como el trastorno por duelo prolongado o el trastorno por duelo complejo persistente. Ambos se diagnostican en caso de una muerte confirmada, y reflejan la dificultad para realizar tareas centradas en la pérdida y de restauración de las funciones cotidianas. Entre sus síntomas, están el anhelo continuo de la persona fallecida, dificultades de identidad para mantener relaciones y establecer vínculos, no poder continuar con la vida, falta de aceptación de la pérdida, evitar los recordatorios sobre el acontecimiento, malestar emocional, preocupación por la persona y la pérdida de sentido (Jordan y Litz, 2014).

Sin embargo, tales síntomas son inapropiados para analizar el malestar de los familiares, amigos y comunidades de las personas desaparecidas, pues parten de la idea de que existe un tiempo determinado para que el duelo se considere patológico, que en el caso de la desaparición puede prolongarse por años debido a la falta de información y de certeza sobre el estado del desaparecido. La temporalidad del duelo también es cuestionable debido a las diferencias culturales sobre la concepción de la muerte y su manejo. A lo largo de la historia, se ha considerado que el duelo ocurre en una serie de etapas lineales y que tiene un punto final si ocurre de forma normal, pero las condiciones de la desaparición impiden el cierre de este proceso. Boss y Carnes (2012) han advertido sobre el riesgo de asignar una patología a los familiares en forma injusta, cuando no se toma en cuenta el tipo de pérdida y el contexto en el que sucede.

Un concepto que puede emplearse para comprender la experiencia de los familiares y de las personas cercanas a las víctimas de desaparición forzada es el de pérdida ambigua: una situación en la que no se sabe si la persona se encuentra viva o muerta. La desaparición forzada se corresponde con el tipo de pérdida ambigua cuando las personas están físicamente ausentes, pero se mantienen psicológicamente presentes (Boss, 2002). Tras la desaparición, las familias viven fuertes cambios en su dinámica. Se producen tensiones entre sus miembros, sobrecarga de responsabilidades, culpa excesiva e incluso la vivencia de una segunda desaparición: la del familiar que sólo se dedica a las labores de búsqueda (Retama y Rojas, 2019). La pérdida ambigua tiene implicaciones en la estructura familiar, ya que los miembros pueden tener percepciones distintas 
respecto a quiénes integran la familia (física o psicológicamente), y esto deriva en una confusión de los roles, en el aplazamiento de decisiones importantes y en la interrupción de las tareas cotidianas o rituales. A su vez, tiene implicaciones psicológicas, pues produce ambivalencia afectiva, malestar emocional, bloqueo de la cognición debido a la incertidumbre y detención del proceso de duelo (Boss, 2004).

De acuerdo con el modelo contextual de estrés familiar (Boss, 2016), se considera que la pérdida ambigua es una situación que provoca estrés crónico, sobre todo porque produce ambigüedad en las fronteras de la familia, es decir, en las percepciones que tienen sus integrantes sobre la persistencia de la persona desaparecida como miembro de la familia. Un alto nivel de ambigüedad incrementa el estrés y afecta la salud mental, aunque ello depende de los recursos individuales, familiares y comunitarios con los que se cuenten. El proceso de afrontamiento de la pérdida ambigua está condicionado por un contexto externo en el que intervienen aspectos institucionales, sociales, culturales y políticos (Boss, 2016).

Heeke y Knaevelsrud (2015) señalan que los familiares de las personas desaparecidas sufren mayor malestar psicológico que las personas en duelo por muertes confirmadas, mayor severidad en los síntomas de depresión y reacciones prolongadas de duelo. Entre los factores de riesgo que contribuyen a presentar problemas de salud mental, se encuentran la exposición a eventos traumáticos, la falta de apoyo social y de rituales de duelo, así como las dinámicas de conflicto familiar. Las consecuencias en la salud mental pueden persistir más de veinte años después de la desaparición o muerte de un familiar en términos de síntomas depresivos, complicaciones del proceso de duelo o síntomas de trastorno por estrés postraumático, como escenas retrospectivas, sustos o entumecimiento (Pérez-Sales, Durán-Pérez y Bacic, 2000).

Para Boss (2007), la pérdida ambigua es un fenómeno relacional y sus causas se encuentran en el contexto. Más que una patología individual, puede considerarse un sufrimiento ocasionado por la incertidumbre crónica, inherente a la situación (Boss, 2016). En estas circunstancias es erróneo diagnosticar a los familiares con trastorno por estrés postraumático, porque la pérdida ambigua produce en el individuo un sufrimiento que tiene un origen social, y el hecho de una desaparición niega de forma implícita la intencionalidad ideológica de la violencia, que garantiza la impunidad, un factor determinante para la prolongación del sufrimiento. A su vez, considerar el problema como individual promueve el uso de tratamientos individuales, mientras que mantener su origen social permite atenderlo a través de intervenciones sociales y comunitarias (Pérez-Sales, Durán-Pérez y Bacic, 2000).

La pérdida ambigua no tiene el mismo impacto en todos los miembros de la familia. Depende de variables individuales, como las atribuciones (por ejemplo, que el hijo andaba en "malos pasos"), las creencias, el nivel de tolerancia a la ambigüedad, la orientación que se tenga para el control o el pensamiento dialéctico, que es la capacidad de mantener en forma simultánea dos ideas opuestas (Boss, 2004). Debido a las diferencias de género socialmente construidas, las 
madres, ante la pérdida de un hijo, pueden ser más vulnerables a la alteración de la salud mental (Kersting y Kroker, 2010). De acuerdo con Maier (2001), la desaparición forzada puede representar para ellas la ruptura de la vida cotidiana y de su identidad, debido a que si se ha construido la feminidad en torno a la maternidad y al cuidado de los otros, se produce un sufrimiento de origen social al que se responde con la búsqueda del hijo en el espacio público, tanto de forma individual como colectiva.

Puesto que aún son pocos los estudios científicos sobre la pérdida ambigua y existe un vacío de conocimiento al respecto, la presente investigación pretende subsanar un poco esa carencia. Sin embargo, su contribución no se limita al estudio de los impactos psicosociales de las desapariciones, sino que busca proporcionar elementos para diseñar intervenciones culturalmente sensibles y para mejorar los tratamientos existentes ofrecidos en los niveles público y privado para atender a las víctimas, en particular a las madres. Por esa razón, el propósito del presente estudio es comprender la experiencia de pérdida ambigua en las madres que tienen hijos víctimas de la desaparición forzada. Se busca identificar los elementos esenciales de este tipo de pérdida, conocer las principales consecuencias psicosociales del acontecimiento y analizar la influencia del contexto sociopolítico en el sufrimiento social de las madres.

\section{Método}

Se realizó un estudio con orientación metodológica de análisis fenomenológico-interpretativo, el cual busca examinar la forma en que las personas otorgan sentido a experiencias vitales importantes. Se asume que las personas actúan en el mundo, reflexionan sobre lo que hacen y que sus acciones tienen consecuencias significativas y existenciales. Se parte de una posición intermedia entre la reconstrucción de la experiencia de la persona en sus propios términos y el empleo de perspectivas teóricas externas para profundizar en la comprensión del fenómeno (Smith, Flowers y Larkin, 2012).

El trabajo de campo se realizó en Ciudad Victoria, Tamaulipas (México). Las participantes fueron seleccionadas de forma propositiva, empleando como criterios de selección el tener uno o más hijos desaparecidos y que hubiera transcurrido al menos un año desde la desaparición. Se realizó el contacto a través de una asociación civil local de familiares de desaparecidos. Los datos se recolectaron en una oficina de la Universidad Autónoma de Tamaulipas.

Participaron cinco mujeres cuyas características socio-demográficas se detallan en la tabla 1. La escolaridad de las madres era diversa: posgrado, licenciatura, carrera técnica, secundaria y primaria no concluida. Sólo una se identificó como activista, aunque todas participaban en una asociación para familiares de personas desaparecidas. Además del hijo desaparecido, tenían entre dos y tres hijos. En la mayoría de los casos, el hijo fue desaparecido en solitario. En todos los casos se negó que los hijos tuvieran cualquier pertenencia o relación con el crimen organizado. 
Tabla 1. Características de las participantes del estudio

\begin{tabular}{|c|c|c|c|c|c|c|}
\hline No. & Edad & $\begin{array}{c}\text { Estado } \\
\text { civil }\end{array}$ & Religión & Ocupación & $\begin{array}{c}\text { Tiempo de la } \\
\text { desaparición }\end{array}$ & $\begin{array}{c}\text { Sexo y edad del hijo } \\
\text { al ser desaparecido }\end{array}$ \\
\hline 1 & 49 & Casada & Católica & Servidora pública & 4 años & Mujer, 25 \\
\hline 2 & 52 & Casada & Católica & Ama de casa & 3 años & Hombre, 27 \\
\hline 3 & 63 & Divorciada & Católica & Vendedora de ropa & 5 años & Hombre, 34 \\
\hline 4 & 35 & Casada & Sin religión & $\begin{array}{c}\text { Empleada en } \\
\text { maquiladora }\end{array}$ & 2 años & Hombre, 15 \\
\hline 5 & 46 & Casada & Cristiana & Ama de casa & 5 años & Mujer, 19 \\
\hline
\end{tabular}

Fuente: elaboración propia.

Se realizaron entrevistas en profundidad mediante una guía con los siguientes ejes: 1) el acontecimiento de la desaparición; 2) la respuesta de las autoridades; 3) la relación con su hijo o hija antes de la desaparición; 4) la vida cotidiana durante la desaparición; y 5) las consecuencias psicosociales de la desaparición. Los ejes incorporaron tres de las categorías que propone Molina (2010) para el estudio de la memoria de personas afectadas por la violencia en un conflicto armado: hechos, sentimientos y afrontamiento. Todas las entrevistas se realizaron en una sola sesión y tuvieron una duración de entre una y dos horas.

Una vez transcritas las entrevistas, el examen se llevó a cabo mediante el procedimiento que describen Smith, Flowers y Larkin (2012) para el análisis fenomenológico interpretativo: 1) lectura de los textos; 2) elaboración de notas descriptivas, lingüísticas y conceptuales; 3) desarrollo de temas emergentes; 4) identificación de relaciones entre los temas emergentes para cada caso y 5) búsqueda de patrones a través de los casos. La interpretación de estos patrones se realizó desde una hermenéutica que buscaba aproximarse a la experiencia de las madres en sus propios términos, pero a la vez que considerara la influencia del contexto sociopolítico en la vivencia de la desaparición. Se realizó una triangulación del análisis a partir de los investigadores mediante reuniones en las que se comparaban y se discutían los patrones identificados, hasta llegar a un acuerdo sobre los más relevantes y su organización. Se presentaron los resultados a cuatro de las participantes para verificar si se aproximaban a su experiencia.

En apego a los estándares éticos de la investigación, antes de realizar las entrevistas a las participantes, se les explicó el propósito del estudio y se les entregó - para que lo firmaran- un consentimiento informado en el que se especificaba que su participación era libre, anónima y revocable, por lo que no se solicitaron datos de identificación de las participantes, sólo algunos sociodemográficos. Se les comentó que podían abandonar la entrevista en el momento que lo desearan y que podían dejar de contestar alguna de las preguntas si ésta les parecía inapropiada o incómoda. Uno de los investigadores con experiencia clínica para brindar contención emocional llevó a cabo las entrevistas, pero ésta no fue necesaria. Una vez transcritas las entrevistas, se eliminaron los audios para mayor protección de las participantes. 


\section{Resultados}

Incertidumbre, ruptura y ausencia

La desaparición es una situación ambigua que implica para las madres vivir con una incertidumbre que tiene múltiples significaciones. En primer lugar, representa el desconocimiento de la localización del hijo y de su estado, es decir, si se encuentra vivo o muerto:

Es desaparecido. No puedo decir que lo perdí, porque hasta ahorita nadie me ha dicho dónde ha estado. Lo traigo desaparecido. No está donde debe estar. Nadie sabe dónde está, eso es una desaparición. (Caso 1)

En segundo lugar, la desaparición se vincula a la falta de información sobre el acontecimiento y los motivos por los cuales su hijo fue desaparecido. Ninguna refirió que el crimen organizado hubiera amenazado previo al suceso; sólo una mencionó haber tenido acceso a testigos de la desaparición y que ésta estaba asociada con la acción de grupos delictivos. A la incertidumbre sobre el origen de la desaparición, se aúna la falta de nueva información que favorezca la localización del hijo. Alimenta la incertidumbre la falta de hallazgos durante el proceso de búsqueda que llevaron a cabo los familiares y las instituciones.

No hemos sabido nada de los tres, de ninguno. Se encontró el carro al mes de que pasó esto. El carro aparece por un lugar y a mi hijo lo retiran por otro. El carro iba rumbo a Ciudad Mante. No hemos tenido respuesta de nada. En cuanto a las autoridades, sí hay papeleo, mucho papeleo, pero en cuanto al resultado, sigue siendo igual: nulo. (Caso 2)

Para las madres, el acontecimiento de la desaparición cambia la vida cotidiana. Es una ruptura de su vida previa. La ausencia física del hijo transforma la manera de habitar el mundo: las madres sufren el malestar de la ausencia y a la vez intentan mantener su presencia psicológica. Inician un proceso de búsqueda mediado por la familia, grupos de víctimas o instituciones públicas: "Pues me dedico al hogar. Ya no salgo ni a caminar. Una vida normal ya no se puede; trato de llevarla, pero siempre vivo con el miedo, con la incertidumbre" (Caso 2).

El alejamiento de los otros

La desaparición ocurre en un contexto sociocultural que configura la experiencia compartida de las madres. En algunos casos, expresan que no han sufrido rechazo o que incluso han recibido muestras de solidaridad. No obstante, puede ocurrir un alejamiento por parte de los otros que obedece a múltiples factores. El distanciamiento puede deberse a que las personas no saben cómo acercarse a la madre y brindarle compañía o apoyo ante la ausencia física de su hijo:

No quiero decir que sea mala la gente, sino que se quedaron sin palabras, de qué decirle a un dolor tan grande como éste, a un problema tan fuerte. Tus amigos ya no saben qué decirte, ya no saben qué decirte. Si 
te dicen una cosa, lo tomas bien, lo tomas a mal; ya no saben ni cómo lo vas a tomar [...] Ahora sí que el que se queda es el que aguantó verte así, y aguantó todo, y sigue siendo tu amigo ahorita, pero los demás se convirtieron en conocidos. (Caso 1)

Sobre las madres puede recaer el juicio social por la forma en que han actuado antes o después de la desaparición. Es posible que reciban críticas por no haber cuidado lo suficiente al hijo como para protegerlo del acto violento, sobre todo cuando es adolescente. Las críticas también suelen destinarse a la forma en que responden a la desaparición, pues en ciertos casos se percibe que la madre no manifiesta el sufrimiento suficiente por su hijo:

Cuando mi hijo desapareció, caí en depresión. Tuve que levantarme y regresar a mi trabajo. La gente decía que me valía lo de mi hijo porque me miraban ir y regresar como si nada, y a lo mejor eso hace que los aleje, porque gente que me conoce antes y ahora es por lo que a lo mejor se imagina, que me ven que voy y vengo, pero solamente yo sé cómo ando. (Caso 4)

El alejamiento de los miembros de la comunidad a la vez se relaciona con la estigmatización de la víctima de desaparición, la cual se manifiesta en la creencia de que "andaba en malos pasos". Sostienen este proceso los discursos gubernamentales y sociales que establecen distinciones entre víctimas "inocentes", "sospechosas" y “culpables", en función de alguna relación, confirmada o supuesta, con el crimen organizado. A partir de ese discurso, la desaparición de la víctima culpable o sospechosa se torna justificada y comprensible, mientras que la desaparición de la víctima inocente se vuelve aún más incierta: no se logra justificar ni se puede comprender por qué ocurrió. Las madres no sólo sufren el daño a la memoria de sus hijos, sino que además las personas pueden alejarse de ellas por temor, como una medida de seguridad, ante la sospecha de supuestos lazos con el crimen organizado:

Las amistades se empiezan a retirar, porque no saben, "seguramente andaba mal" o mil supuestos, que la gente no sabe, y eso ha hecho mucho daño a los que padecemos esto, porque te quedas solo, incluso la misma familia se aleja, se aleja de uno [...]. No se ha llegado a entender que desaparecidos hay de todo tipo. A lo mejor sí, efectivamente andaban trabajando en algo ilícito, pero otros no. (Caso 1)

\section{La ineficiencia del Estado}

En las narraciones de las madres se expresa un malestar por la ineficiencia de las instituciones de procuración de justicia. Dicha ineficiencia se manifiesta desde el momento de la denuncia, pues en ocasiones no se inicia la búsqueda de inmediato. Argumentan los agentes de esas instituciones que tiene que transcurrir un tiempo determinado para verificar si se trata de un extravío, de una desaparición o de una salida voluntaria del hogar: 
Fui a la ministerial a poner la denuncia por la desaparición. Me dijeron que tenían que pasar setenta y dos horas, que a lo mejor se había ido con el novio, y yo "¡Claro que no! ¿Cómo crees que mi hija se va a ir con el novio?" Ella no era de andarse en la calle ni andarse quedando con amigas ni nada. A ella tuvo que haberle pasado algo bastante grueso para que no haya llegado a casa. (Caso 5)

El proceso de búsqueda requiere un peregrinaje institucional para localizar a sus hijos entre las víctimas de otros delitos, acudir a los servicios forenses y solicitar la colaboración de las procuradurías de otras entidades federativas debido a la posibilidad de que su hijo haya sido trasladado a otro sitio. Una de las participantes, quien se identifica como activista, señala la ausencia de protocolos especializados para la investigación de casos de desaparición o la aplicación inadecuada de los procedimientos existentes. La percepción de ineficiencia institucional se fortalece conforme transcurre el tiempo y no se localiza al familiar ni se obtienen pistas o nuevos indicios, a pesar del peregrinaje institucional:

Ha sido un largo camino, con muchos detalles. Inmediatamente se puso la denuncia, inmediatamente se buscó. En aquella época, en 2012, nadie estábamos preparados. La Procuraduría tampoco estaba preparada. No había un protocolo de búsqueda, eh, no había instrumentos que apoyaran la localización de señales de celular, los procedimientos para solicitar apoyo de investigación, no había [...]. Se construyó sobre el dolor, una serie de cosas que ahora se tienen. Pero, bueno, ha costado mucho y ella no está, sigue sin estar, sigue sin haber información de nada. (Caso 1)

No sólo existen quejas por la incompetencia en la investigación, sino también por la actitud de las autoridades ante los casos individuales. Una situación que indigna a las madres es que el personal de las instituciones de justicia les pregunte acerca de lo que han averiguado o soliciten nueva información con base en sus búsquedas individuales. Se percibe que la búsqueda no se realiza de manera apropiada no sólo por la ineficiencia en los procedimientos, sino también por la falta de preocupación o anhelo de encontrar a las personas desaparecidas, a diferencia de lo que ocurre en los familiares que realizan una búsqueda personal fomentada por sus afectos. En contraste, la búsqueda que hacen las instituciones se siente pasiva, lenta, desapegada, burocratizada e incluso simulada:

A raíz de que pusimos la denuncia, fuimos y fuimos. Íbamos casi casi a diario. Bueno, ya al último, la gente que estaba según en la búsqueda, nos preguntaba qué era lo que sabíamos nosotros, y ellos, hasta la fecha, no me han dado ninguna pista, nada, nada, ni el detalle más mínimo. Nosotros hemos hecho, pues, casi todo. Cuando mi hijo desapareció, se repartieron volantes, se puso reporte en radio, en televisión, lonas, mantas con la fotografía de mi hijo con teléfonos y hasta con la dirección de donde salió mi hijo, que es la de mi casa, con la esperanza de que si alguien lo veía o sabía algo de él, pues nos llamaran, y no. (Caso 4) 
La respuesta ineficiente de las instituciones de justicia en diferentes momentos de la búsqueda, así como la falta de un interés genuino en que la víctima aparezca, promueven la participación de las madres en el proceso de búsqueda de su hijo, en forma paralela o incluso en una relación de tensión y cooperación con las instituciones. En un primer nivel, las madres inician la búsqueda por medio de reportes en medios de comunicación, repartiendo volantes o colocando carteles con los datos del hijo desaparecido, incluso mediante páginas de internet para obtener información relacionada con la desaparición. Después realizan búsquedas físicas que, aunque implican riesgos para su seguridad, al realizarlas en colectivo favorecen la expresión social del malestar emocional y la compañía solidaria de otras madres.

\section{Mantener el vínculo con el hijo desaparecido}

La ausencia física del hijo se contrarresta con el trabajo emocional de la madre para asegurar su presencia psicológica en la cotidianidad. Dicho proceso se ha denominado mantenimiento del vínculo y consiste en diversas prácticas, sentimientos y significados que permiten a la madre conservar una relación afectiva a pesar de la desaparición. En este proceso convergen diversas temporalidades, pues se articulan el pasado, el presente y el futuro para conservar su presencia psicológica.

La dimensión del pasado se manifiesta en la memoria que construye la madre sobre la vida previa a la desaparición. Todas las participantes mencionaron que mantenían una relación cercana con su hijo, independientemente de que vivieran juntos o no en el momento del suceso. Además, intentan construir una imagen positiva del hijo, que contradice las supuestas asociaciones que se establecen entre las víctimas de desaparición con el crimen organizado. Por medio de la memoria no sólo se construye un recuerdo entrañable de la historia compartida, sino que además se respalda la identidad moral del hijo ante los otros:

Los fines de semana se iba a tomar un café o salía con los muchachos con los que andaba, pero diversión sana. Él era [...]. Voy a hablar en presente [...]. Él es muy precavido para sus amistades, de hecho, no cualquiera se metía a la casa, ellos sí; yo los veía como si fueran mis hijos. Pero yo al que puedo meter las manos al fuego es por mi hijo [...] Iba a la iglesia, casi siempre estaba con nosotros. (Caso 2)

En la dimensión del presente tienen lugar las principales acciones cotidianas de mantenimiento del vínculo. En el nivel lingüístico, las madres suelen hablar en tiempo presente de sus hijos, o alternar el tiempo presente con el tiempo pasado. Eso revela la tensión entre la ausencia física que contribuye a pensar que el tiempo del hijo ha concluido, que su historia está cerrada y ubicada en el pasado, y la presencia psicológica, que favorece la noción de que su historia aún no ha culminado y se contempla un escenario donde sigue con vida.

Las madres realizan diversos actos para recordar al hijo, como escuchar su música, contemplar sus fotografías, contar sus cumpleaños o los días de su des- 
aparición. El ritual con el que pueden acercarse a sus hijos de manera más íntima es hablar con ellos, lo que implica recordar episodios previos de la historia compartida, narrar lo que hacen en la cotidianidad, compartir el proceso de la búsqueda, expresar el cariño que sienten hacia él o cómo se sienten con su ausencia física. De manera implícita, el ritual está ligado a la fe y a la confianza de que el hijo se encuentre vivo, pues no es el habla dirigida a alguien de quien se tiene certeza sobre su muerte. La conversación les permite sentir que su hijo está junto a ellas, presente de forma psicológica a pesar de la incertidumbre ligada a la desaparición:

Cuando salgo le digo "Ya me voy"; cuando llego le digo "Ya llegué". Igual cuando voy a operativo, este, pues igual, "Voy a buscarte a ver si estás allí"; llego y "Pues no te encontré, dime dónde puedes estar". (Caso 1)

En la dimensión del futuro, las madres expresan un sentido de esperanza para que el hijo aparezca o regrese con vida. Están conscientes de la posibilidad de que esté muerto o de que no aparezcan sus restos, pero la esperanza entraña priorizar el anhelo de su retorno y la restauración del orden moral, es decir, que su hijo pueda recuperar su libertad, continuar con su proyecto de vida y reintegrarse a la comunidad. La esperanza se observa en el acto de guardar las pertenencias del hijo o conservar su espacio dentro del hogar. Las madres expresan que continuarán esperando su regreso:

Tengo su ropa en la casa, son quince camisas que tengo. Están guardadas $y$, de repente, toca que ando en la ropa de mi closet y la miro y digo: "Aquí está tu ropa". Me contesto yo misma, pero nada más eso hago. (Caso 3)

Añoro que regrese. Me paro en el portón, viendo pa' todos lados. A pesar del tiempo, me imagino que va a llegar. Lo he soñado, que regresa. He sentido su aliento, que me besa. No he perdido la esperanza. Eso es lo que anhelo y añoro: verlo. (Caso 2)

\section{El malestar de la desaparición}

El malestar se manifiesta en los relatos de las madres a través de múltiples formas de expresión. En primer lugar, emplean un lenguaje íntimo del sufrimiento, en el que vivir la desaparición se simboliza con términos como un infierno o un martirio, que aluden a la pena continua, el sufrimiento del cual no pueden salir. Predomina en la experiencia social de las madres el sentimiento de un dolor que no se va, que puede tener diferentes ritmos e intensidades: hay quienes perciben que el dolor es el mismo desde el día que su hijo desapareció. Otras personas sienten que se agrava con el tiempo y hay madres que mencionan que el dolor es intermitente, porque en algunos días es de mayor intensidad:

Es un infierno, la verdad. El no verla no tiene ningún fin [...]. Se levanta uno con el mismo pensamiento de ella y se acuesta uno con el mismo 
pensamiento [...]. Anda uno caminando, anda uno trabajando, como muerto en vida. (Caso 1)

No se trata sólo del sentimiento de dolor, sino que también se pierde la tranquilidad y piensan en sus hijos desaparecidos todos los días, todo el tiempo. Se levantan y se acuestan con el mismo pensamiento: dónde estará, qué le han hecho, qué está haciendo, en qué estado se encuentra. Así mismo, el dolor establece un distanciamiento con los otros, a causa de que las madres se mantienen inmersas en su dolor, incluso al grado de no percibir el de los demás. Creen que sienten un dolor diferente por ser madres y experimentan una sensación de aislamiento, puesto que consideran que los demás no pueden comprender lo que ellas sienten ni vivir el dolor con la misma intensidad que ellas:

Cada que amanece el día, estás pensando dónde está, que en algún lugar está, pero dónde, no se puede, por más que pienses y pienses, no sabes nada. $O$ te acuestas, e igual a la hora que despiertas, por más que quieras no hacer eso, lo piensas. No hay un día que yo no haya llorado. Es un sentimiento muy difícil que te entiendan, tu familia. Sólo uno que lo vive, lo siente, y más como madre. (Caso 3)

La experiencia del dolor materno es el elemento central en su lenguaje íntimo del sufrimiento que sólo otras madres en su misma situación pueden compartir y entender. En algunos casos, se alude a la sensación de "vivir en automático". Esto significa que las madres siguen viviendo, hacen su rutina cotidiana, pero han perdido la vitalidad, la alegría o la satisfacción de vivir. Continúan viviendo por obligación, es decir, porque tienen que hacerlo, no porque quieran hacerlo u obtengan placer en ello. Existe la sensación de que su mundo se ha transformado por la ausencia física del hijo, que ya no tiene el mismo valor y que la existencia ya no tiene el mismo sentido. En tales circunstancias, la vida se vuelve pura repetición, automatismo de supervivencia.

Y de mi día sin mi hijo, pues lo vivo porque lo tengo que vivir: me levanto, atiendo a mis hijos que van a la escuela, me voy a mi trabajo, mi esposo se encarga igual de recogerlos, yo estoy en mi trabajo hasta las dos o tres de la tarde, si no hasta la tarde, hasta las siete. Llego a mi casa y, pues si hay algo que hacer de la casa, de lavar trastes, dar de cenar, arreglar uniformes, lo hago, y me acuesto, pero para mí todos los días son iguales, desde el momento en que mi hijo desapareció. (Caso 4)

En segundo lugar recurren al discurso psiquiátrico para simbolizar el malestar. Hacen referencia a la vivencia de sintomatología psicológica, en algunos casos a síntomas de ansiedad, de angustia o incluso de estrés postraumático. En otros casos refieren de manera explícita la depresión y enumeran síntomas como la alteración del sueño, la alimentación o el peso corporal. Su sintomatología física incluye contracturas, caída del cabello, alteración de la presión arterial, o enfermedades específicas, como herpes o displasias. A veces se refieren a esta problemática de forma general, como un estado de debilidad, de malestar 
físico, de enfermedad constante y de visitas recurrentes al médico. En ciertos casos, las madres asisten a los servicios de salud, sobre todo para atender síntomas físicos. Sólo se atienden los síntomas psicológicos cuando tienen un mayor nivel de severidad o cuando les provocan incapacidad:

Estaba en un quebranto tremendo [...]. Me bajé más de seis kilos en menos de un mes, y como mi cuerpo obviamente lo resintió, mal, muy mal, tuve que ir al médico y todo. Pensé, "Bueno, estoy mal, porque yo me puedo dejar morir, no como, entro en depresión, y todo ese rollo", pues ya sé las formas, las tácticas del diablo, siempre viejas, las mismas de siempre, trae un espíritu de depresión sobre una vida, hasta llegar al momento en que se quiten la vida. (Caso 5)

En tercer lugar se encuentra un lenguaje centrado en las emociones, a partir del cual se busca clasificar la vivencia del sujeto en determinadas categorías afectivas. La emoción que viven las madres con mayor intensidad es la tristeza y se manifiesta en el llanto constante, en la sensibilidad hacia las referencias a la desaparición y en la pérdida de la alegría. Mencionan que han perdido el deseo de vivir, acusan una sensación de vacío o falta de sentido e incluso algunas han contemplado la idea de morir, aunque ninguna mencionó ideaciones o intentos suicidas:

Es mucha tristeza porque es algo que ni olvidas ni superas, y es un dolor que allí está [...]. Mi dolor es igual que el de la primera vez, desde el primer día que supe que no estaba mi hijo, que no lo encontramos, que nadie sabía de él, es mucha tristeza. A veces ni las cosas que a mí me deberían de dar alegría o felicidad, siento que ya no les tomo la importancia que les daba antes ni la importancia que se les debe dar. (Caso 4)

A diferencia de la tristeza, que es una emoción experimentada de forma intensa en todos los casos, se mencionaron otras emociones con mayor variabilidad en su expresión; es decir, las madres reportaron niveles tanto bajos como elevados de las siguientes emociones. Expresaron sentir soledad ante la ausencia del hijo, aun cuando se encuentren acompañadas por sus familiares. Señalaron sentirse culpables, ya sea por no haber evitado la desaparición o por no haberlos cuidado suficiente, pero también por las cosas que no pudieron hacer con sus hijos. Por último, mencionaron sentir furia, amargura e impotencia ante la desaparición. Hay indignación, porque ya sea que se trate de la desaparición de un hijo o de una hija, tanto las autoridades como la sociedad suelen juzgarlos de manera moral. Si se trata de un varón, "seguramente andaba mal"; si se trata de una mujer, "se fue con el novio". Las madres intentan defender la honra de sus hijos frente a las instituciones y ante la comunidad y mantener el vínculo con ellos a través de su presencia psicológica. Puede decirse que las madres de hijos desaparecidos viven un entramado emocional complejo, un sufrimiento social en el que predomina la tristeza y que puede intensificarse hasta perder el deseo de vivir, con una sensación de vacío o incluso se puede contemplar la posibilidad de la muerte como último remedio. 


\section{Discusión}

El sufrimiento social que experimentan las madres de personas desaparecidas está enmarcado por un contexto de corrupción, impunidad e ineficacia de las instituciones del Estado que limita el proceso de investigación de los casos y contribuye a la prolongación de la incertidumbre y el sufrimiento (Zorio, 2011). Ante tales circunstancias, las madres emprenden la búsqueda de sus hijos de forma personal o acompañada de organizaciones civiles, ante la desconfianza que tienen en el Estado. Sin embargo, dicha desconfianza no es inherente a las instituciones, sino que se va gestando en el proceso de búsqueda, en momentos clave como la denuncia, la búsqueda en fosas clandestinas, el intento de colaboración con otras procuradurías estatales y, de manera primordial, por la percepción que tienen de que la búsqueda está burocratizada y de que sus hijos se reducen a números y expedientes. El cuidado de su propia salud pasa a segundo plano, pues lo esencial para las madres es la recuperación de su hijo, vivo o muerto, más que la obtención de una verdad jurídica, histórica o clínica (Palma, 2016).

La ausencia del cuerpo impide las ceremonias tradicionales que se realizan en caso de una muerte verificada. La ambigüedad inherente al suceso limita la respuesta de los miembros de sus redes sociales, quienes se alejan al no saber cómo lidiar con el dolor de las madres (Robins, 2016). El aislamiento de las madres también puede deberse a la estigmatización del desaparecido como supuesto miembro del crimen organizado. En el contexto local, pensar que las desapariciones sólo ocurren por la vinculación con grupos criminales, puede contribuir a mantener la creencia de que el mundo es justo y a que las personas se sientan menos vulnerables al no pertenecer a dichas organizaciones.

El aislamiento de las madres se exacerba en un ámbito en el que el Estado promueve el silencio, no reconoce de forma adecuada las desapariciones ni favorece la memoria colectiva sobre estos acontecimientos. De acuerdo con el modelo contextual de estrés familiar (Boss, 2016), puede señalarse que la vinculación entre el Estado y el crimen organizado, la ineficiencia institucional, la estigmatización de las víctimas y la falta de rituales culturales ante la desaparición, son los elementos más importantes del contexto externo que condicionan el manejo del suceso.

La categoría de pérdida ambigua es pertinente para aproximarse a la experiencia de sufrimiento social y dolor individual derivado de la desaparición forzada. Entre sus elementos esenciales, se encuentran la incertidumbre (sobre el estado del hijo, su localización, el motivo de la desaparición o los avances en la investigación) y la ruptura del mundo cotidiano ante su ausencia física. Un hallazgo del estudio es que las madres fomentan la presencia psicológica del hijo a través de mecanismos de mantenimiento del vínculo en tres dimensiones temporales: la imagen positiva del hijo (pasado), el uso de tiempos verbales, actos para recordarlo y hablar con él (presente) y conservar la esperanza (futuro). Y aunque la esperanza puede exacerbar el malestar al limitar el proceso de cierre (Wayland, Maple, McKay y Glassock, 2015), también permite el mantenimiento del vínculo. 
Si bien mantener el vínculo permite la presencia psicológica del hijo, su ausencia física provoca un malestar que se expresa de múltiples formas. Desde un lenguaje local de malestar (Robins, 2016), se vive como un infierno o martirio que produce dolor, se piensa de manera continua en el hijo y se pierde el sentido o el placer en la vida cotidiana. Está presente el discurso psiquiátrico que indica la presencia de sintomatología de tipo depresiva o ansiosa, así como diversos problemas de salud. Mediante el lenguaje emocional, se encontró un entramado afectivo en el que predominan la tristeza y la desesperanza.

Tal como se ha reportado en estudios previos, el malestar que expresan las madres de hijos desaparecidos se asocia con sintomatología depresiva o duelo prolongado (Boss, 2016; Heeke y Knaevelsrud, 2015). En el presente estudio las participantes no expresaron con frecuencia síntomas de estrés postraumático, en contraste con estudios previos (Heeke, Stammel, Heinrich y Knaevelsrud, 2017; Heeke, Stammel y Knaevelsrud, 2015). Más bien, la experiencia de la desaparición de un hijo está polarizada: por un lado, se encuentra el mantenimiento de un vínculo positivo con el fin de asegurar la presencia psicológica del hijo y, por el otro, se vive el malestar asociado con la ausencia física. Pueden considerarse como dos dimensiones interrelacionadas, porque mantener el vínculo permite la aproximación simbólica con el hijo y otorga intencionalidad y propósito a una vida cotidiana que se ha transformado desde la desaparición. El malestar representa el retorno continuo de la ausencia y el sufrimiento que promueve la construcción de un sentido trágico o melancólico de la vida. Ambas dimensiones parecen complementarse en una relación dialéctica, a través de la cual la persona continúa existiendo, pero se trata de una existencia dolorosa.

Aunque las madres salen de manera individual o en colectivo al espacio público para buscar a sus hijos ante la inoperancia del Estado, eso no implica necesariamente su transformación en activistas o la reconstrucción de su identidad de género, pues el cuidado de los otros no sólo sigue siendo prioritario para la búsqueda, sino que también lo es para el mantenimiento del hogar y el cumplimiento de funciones de crianza en la cotidianidad. La necesidad de mantener el vínculo con el hijo desaparecido es mayor en el caso de las madres, puesto que la cultura ha promovido la construcción de su identidad en torno a la maternidad. Por eso las madres perciben una diferencia con respecto a los otros miembros de la familia: tienen dificultad para desligarse de modo afectivo del hijo desaparecido, debido a la intensidad del dolor y tienen renuencia a continuar con su proyecto de vida individual y a olvidarse de su hijo. Esta disposición las pone en una situación que vulnera la salud mental, sobre todo porque se prioriza el cuidado de los otros sobre el cuidado de sí mismas y porque el sufrimiento se enmarca en la esfera de lo individual, aunque en realidad es eminentemente social.

\section{Conclusiones}

El sufrimiento derivado de la pérdida ambigua se origina y se desarrolla en el ámbito de lo social. La experiencia de dicha pérdida está influida por el contexto 
de violencia, corrupción e impunidad, y por la sistemática ineficiencia de las instituciones que lleva a las madres a emprender la búsqueda paralela de sus hijos, de manera individual o colectiva. El desarrollo de sintomatología psicológica en las víctimas indirectas de la desaparición suele diagnosticarse como un problema individual. Sin embargo, resulta problemático asignar una patología individual a un sufrimiento de origen social (Pérez-Sales, Durán Pérez y Bacic, 2000). Es necesario que el Estado reconozca su participación en el origen y en la continuidad del sufrimiento y que sus intervenciones incorporen la dimensión psicosocial y comunitaria. Incluso es fundamental que las intervenciones traten de modificar el discurso sobre la desaparición en el contexto local (Robins, 2016).

Entre las limitaciones del estudio, se encuentra la homogeneidad de las participantes, por lo que se requiere incorporar la perspectiva de otros grupos de madres de personas desaparecidas, como el caso de mujeres activistas que cuentan con mayor empoderamiento y recursos para manejar la desaparición. También están ausentes las voces de las madres más vulnerables, quienes no han acudido a las instituciones tras la desaparición, o de las que han sufrido al mismo tiempo desplazamiento forzado, o de las que temen acudir a las instituciones porque han recibido amenazas o porque sus familiares han sido miembros del crimen organizado. Es necesario incorporar las voces de otros miembros de la familia para identificar las diferencias en el malestar y en el afrontamiento, así como los cambios en la estructura e interacción familiar. También es importante analizar con mayor profundidad las diferencias en cuanto al dolor que experimentan las madres ante la pérdida de un hijo, de una hija, de una hija o un hijo únicos, cuando el hijo o la hija tienen hijos, y si estas diferencias influyen en la forma de buscarlos.

Una de las características de la pérdida ambigua es la existencia simultánea de un vínculo con el hijo y de malestar emocional. Las variaciones en la intensidad del vínculo y del sufrimiento son susceptibles de modificarse a través del tiempo, pero se requieren nuevos estudios para identificar los elementos que promueven el cambio. Por último, la pérdida ambigua ante la desaparición de un hijo es una experiencia más social que individual; lleva a pensar en el afrontamiento en colectivo, un aspecto central porque crea procesos de significación y de agencia que van más allá de lo individual. Se recomienda investigar al respecto, considerando su potencial utilidad para las personas y las familias que experimentan la pérdida ambigua tras la desaparición forzada de un ser querido.

\section{Referencias}

Aguayo, S. (2015). De Tlatelolco a Ayotzinapa. Las violencias del Estado. México: Ediciones Proceso.

Benjamin, W. (1991). Para una crítica de la violencia y otros ensayos. Madrid: Taurus.

Boss, P. (2002). Ambiguous loss in families of the missing. The Lancet, 360(12), 39-40. doi: 10.1016/s0140-6736(02)11815-0 
Boss, P. (2004). Ambiguous loss research, theory, and practice: reflections after 9/11. Journal of Marriage and Family, 66(8), 551-566. doi:10.2307/3600212

Boss, P. (2007). Ambiguous loss theory: challenges for scholars and practitioners. Family Relations, 56(4), 105-111. doi:10.1111/j.1741-3729.2007.00444.x

Boss, P. (2016). The context and process of theory development: the story of ambiguous loss. Journal of Family Theory \& Review, 8(9), 269-286. doi:10.1111/jftr.12152

Boss, P., y Carnes, D. (2012). The myth of closure. Family Process, 51(4), 456469. doi: $10.1111 /$ famp. 12005

Comisión Nacional de Búsqueda. (2020). Búsqueda, identificación y registro de personas desaparecidas. México: Gobierno de México. Recuperado de https://www.gob.mx/cms/uploads/attachment/file/568163/CNB_13_julio_2020_informe_fosas.pdf

Comisión Nacional de los Derechos Humanos. (2017). Informe especial de la Comisión Nacional de los Derechos Humanos sobre desaparición de personas y fosas clandestinas en México. México: Autor. Recuperado de https: / /www. cndh.org.mx/sites/default/files/doc/Informes/Especiales/InformeEspecial_20170406.pdf

Correa-Cabrera, G. (2017). Militarización y violencia en Tamaulipas. En R. Benítez y S. Aguayo (eds.), Atlas de la seguridad y la defensa de México 2016 (pp. 181-189). México: CASEDE.

Correa-Cabrera, G. (2018). Los Zetas Inc. México: Temas de Hoy.

Deotté, J. (2015). México: el desafío estético de la desaparición forzada. Cultura-Hombre-Sociedad, 25(1), 33-45. doi:10.7770/cuhso-v2sn1-art900

Enciso, F. (2016). Los reclamos de justicia de las víctimas como política de Estado. El daño social de las regulaciones sobre drogas en México. México: Instituto Belisario Domínguez y Senado de la República.

Gatti, G. (2011). El lenguaje de las víctimas: silencios (ruidosos) y parodias (serias) para hablar (sin hacerlo) de la desaparición forzada de personas. Universitas Humanística, 72(2), 89-109. Recuperado de https://revistas.javeriana.edu.co/index.php/univhumanistica/article/view/2148

Gatti, G. (2017). Prolegómeno. Para un concepto científico de desaparición. En G. Gatti (Ed.), Desapariciones. Usos locales, circulaciones globales (pp. 13-32). Bogotá: Siglo del Hombre.

Gatti, G., e Irazuzta, I. (2019). Diario de la desaparición mexicana. Entre el precedente y el exceso. Disparidades. Revista de Antropología, 74(2), 1-14. doi: 10.3989/dra.2019.02.019

Gibler, J. (2012). Morir en México. Oaxaca: Sur+Ediciones.

Heeke, C., y Knaevelsrud, C. (2015). Uneindeutiger Verlust Psychopathologische und psychosoziale Konsequenzen im Kontext gewaltsamer Konflikte. Der Nervenarzt, 86(7), 826-832. Recuperado de http://dx.doi.org/10.1007/ s00115-014-4234-0

Heeke, C., Stammel, N., Heinrich, M., y Knaevelsrud, C. (2017). Conflict-related trauma and bereavement: exploring differential symptom profiles of prolonged grief and posttraumatic stress disorder. BMC Psychiatry, 17(1), 118-128. doi: 10.1186/s12888-017-1286-2 
Heeke, C., Stammel, N., y Knaevelsrud, C. (2015). When hope and grief intersect: rates and risks of prolonged grief disorder among bereaved individuals and relatives of disappeared persons in Colombia. Journal of Affective disorders, 173(1), 59-64. doi: 10.1016/j.jad.2014.10.038

Jordan, A., y Litz, B. (2014). Prolonged grief disorder: diagnostic, assessment, and treatment considerations. Professional Psychology: Research and Practice, 45(3), 180-187. doi: 10.1037/a0036836

Kersting, A., y Kroker, K. (2010). Prolonged grief as a distinct disorder, specifically affecting female health. Archives of Women's Mental Health, 13(1), 27-28. doi: 10.1007/s00737-009-0112-3

Maier, E. (2001). Las madres de los desaparecidos. ¿Un nuevo mito materno en América Latina? México: Universidad Autónoma Metropolitana, El Colegio de la Frontera Norte y La Jornada Ediciones.

Molina, N. (2010). Reconstrucción de memoria en historias de vida. Efectos políticos y terapéuticos. Revista de Estudios Sociales, 36, 64-75. doi: 10.7440/ res36.2010.05

Observatorio sobre Desaparición e Impunidad (ODI). (2019). Informe comparado sobre eventos de desaparición. "Nuevo León, Coahuila y Tamaulipas (Región Noroeste)". Recuperado de https://bit.ly/2K6XR6p

Olalde, K. (2015). Marcos de duelo en la guerra contra el narcotráfico en México. Política y Cultura, 44, 57-77. Recuperado de http://www.scielo.org. mx/scielo.php?script=sci_arttext\&pid=S0188-77422015000200004

Organización de Naciones Unidas. (2006). Convención Internacional para la protección de todas las personas contra las desapariciones forzadas. Recuperado de https://www.ohchr.org/sp/professionalinterest/pages/conventionced.aspx

Osorno, D. E. (2017). La guerra de los Zetas. Viaje por la frontera de la necropolítica. México: Penguin Random House.

Padgett, H. (2016). Tamaulipas. La casta de los narcogobernadores: un eastern mexicano. México: Ediciones Urano.

Palma, C. (2016). La desaparición forzada: una verdad caleidoscópica. Desde el Jardín de Freud, 16 (1), 187-212. doi: 10.15446/dfj.n16.58163

Pérez-Sales, P., Durán-Pérez, T., y Bacic, R. (2000). Long-term psychosocial consequences in first-degree relatives of people detained-disappeared or executed for political reasons in Chile. Psicothema, 12(1), 109-116. Recuperado de http: / / www.psicothema.com/psicothema. asp?id=404

Ravelo, R. (2013). Zetas. La franquicia criminal. México: Ediciones B.

Retama, M., y Rojas, M. (2019). La desaparición de personas como forma de violencia y sus efectos. Revista mexicana de ciencias penales (8), 111-126. Recuperado de http://revistaciencias.inacipe.gob.mx/index.php/02/article/view/80/81

Robins, S. (2016). Discursive approaches to ambiguous loss: theorizing community-based therapy after enforced disappearance. Journal of Family Theory \& Review, 8(9), 308-323. doi: 10.1111/jftr.12148 
Robledo, C. (2016). Genealogía e historia no resuelta de la desaparición forzada en México. Revista de Ciencias Sociales, 55 (5), 93-114. doi: 10.17141/ iconos.55.2016.1854

Schedler, A. (2018). En la niebla de la guerra. Los ciudadanos ante la violencia criminal organizada. México: CIDE.

Smith, J., Flowers, P., y Larkin, M. (2012). Interpretative Phenomenological Analysis. Theory, Method and Research. Londres: Sage.

Wayland, S., Maple, M., McKay, K., y Glassock, G. (2015). Holding on to hope: a review of the literature exploring missing persons, hope and ambiguous loss. Deathstudies, 40(1), 54-60. doi: 10.1080/07481187.2015.1068245

Zárate, A., y López, A. (2017). Estudio sobre la violencia en Tamaulipas: diagnóstico y acciones de respuesta. En F. Enciso (ed.), Violencia y paz. Diagnóstico y propuestas para México (pp. 397-425). México: COLMEX, CNDH y Senado de la República.

Zorio, S. (2011). El dolor por un muerto-vivo. Una lectura freudiana del duelo en los casos de desaparición forzada. Desde el Jardín de Freud, 11(1), 251-266. Recuperado de https://revistas.unal.edu.co/index.php/jardin/ article/view/27261 